\title{
Plasma Particle Trajectory Study for a Device Combining Hollow Cathode Discharge and Non-Adiabatic Trap*)
}

\author{
Shuya IWATA, Ryo MATSUMOTO, Kojiro SEKIGUCHI, Takahiro URANO, Gaku SAKAI, \\ Toshiki TAKAHASHI and Hiromu MOMOTA ${ }^{1)}$ \\ Graduate School of Science and Technology, Gunma University, Kiryu 376-8515, Japan \\ ${ }^{1)}$ National Institute for Fusion Science, Toki 509-5292, Japan
}

(Received 18 September 2018 / Accepted 19 November 2018)

The ion trajectory in a hollow cathode discharge plasma to which a magnetic field combined a solenoid and a Helmholtz coil is applied is analyzed, and the ion energy distribution at the cathode or the end of the device is examined. It was shown that the ratio of particles lost at the end of the device and those lost during collision with the cathode can be changed by varying the length of the cylindrical cathode.

(C) 2019 The Japan Society of Plasma Science and Nuclear Fusion Research

Keywords: non-adiabatic trap, Helmholtz coil, hollow cathode, particle-tracking calculation, secondary electron emission

DOI: $10.1585 /$ pfr. 14.2406026

\section{Introduction}

An open-ended plasma can be applied to extract an ion beam from its magnetic force line structure. Ion beams have various uses, some of which have been studied for application in energy production methods such as nuclear fusion.

In recent years, research is being done for the application of ion beams in medical treatment, e.g., in the development of neutron sources for boron neutron capture therapy [1], which is accomplished by utilizing the neutrons generated as a product of nuclear fusion in a deuterium beam. In this application, an ion source is installed facing an electrostatic accelerator, the deuterium beam is confined by a linear electrostatic trap, and the generation of neutrons by the D-D reaction is proposed. In this proposal, the key is the plasma source by cylindrical hollow cathode discharge to which the magnetic field of the solenoid and Helmholtz coil is applied. The hollow cathode can increase the ionization efficiency of the neutral gas by the reciprocating motion of the electrons trapped between the cathodes, so that a relatively high plasma density can be obtained [2]. By incorporating a special magnetic field structure that is generated by a solenoid and a Helmholtz coil, it is possible to control the secondary electron emission and obtain plasma of a higher density. However, this method is yet to be experimentally verified.

To obtain guidelines that need to be applied for future experimental studies, we analyzed the trajectories of deuterium ions in a hollow cathode to which a magnetic field was applied. We obtained the energy distribution of deuterium ions during collision with the cathode, when they

author'se-mail: t-tak@gunma-u.ac.jp

*) This article is based on the presentation at the 12th International Conference on Open Magnetic Systems for Plasma Confinement (OS2018). reached the end of their axial movement. The aim of this study is to analyze the influence that the properties of the ion beam may have on the ion extraction and secondary electron emission processes relevant for the research into boron neutron capture therapy. In addition, it can be said that it is also the first stage to develop this research, advance the development of Monte Carlo code, and enable self-consistent analysis.

\section{Calculation Model}

We calculated the trajectory of charged particles in a device that combines a hollow cathode, a solenoid coil, and a Helmholtz coil, as presented in Fig. 1 and Table 1. The cathode is set to the ground potential and a voltage of $200 \mathrm{~V}$ is applied to the anode. The current flowing through the Helmholtz coil is adjusted so that it cancels with the central magnetic field generated by the solenoid

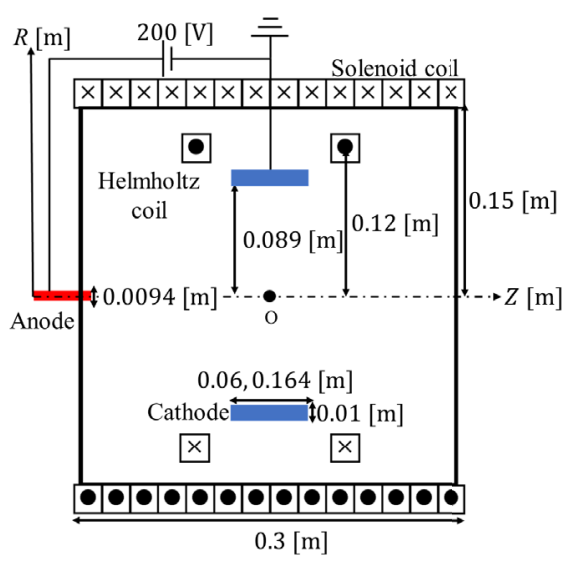

Fig. 1 Device structure. 
Table 1 Device parameters.

\begin{tabular}{c|c}
\hline Solenoid coil turns & $50\left[\mathrm{~m}^{-1}\right]$ \\
\hline Solenoid coil current, $I_{\mathrm{s}}$ & $0.1 \leq I_{\mathrm{s}} \leq 10.0[\mathrm{kA}]$ \\
\hline Helmholtz coil current, $I_{\mathrm{h}}$ & $0.572 \leq I_{\mathrm{h}} \leq 57.2[\mathrm{kA}]$ \\
\hline Confinement region $(R, Z)$ & $0.0 \leq R \leq 0.15[\mathrm{~m}]$ \\
& $-0.15 \leq Z \leq 0.15[\mathrm{~m}]$ \\
\hline
\end{tabular}

coil, shown at the point o in Fig. 1. Calculations were performed by using a two-dimensional cylindrical coordinate system and assuming axisymmetry.

The electric field generated by the electrode will be described hereafter. Placing the anode and the cathode, and using the Poisson's equation

$$
\Delta \phi(R, Z)=-\frac{1}{\varepsilon_{0}} \rho(R, Z),
$$

is solved by Jacobi method to obtain electrostatic potential. Where, $\varepsilon_{0}$ is the permittivity of vacuum. At the boundary of the calculation region, we employ the Neumann condition, which sets the derivative of the electrostatic potential in the direction normal to the boundary to zero. The electric field in the device is determined by a numerical differentiation of the obtained electrostatic potential by using the relation

$$
\mathbf{E}=-\nabla \phi
$$

The charge density, $\rho$ is attained by numerically modeling the electron and ion densities in the device. The charge density distribution in the vicinity of the cathode is changed by adjusting the peak and the value of the full width at half maximum, as described in von Engel's textbook [3].

Our calculations were performed for cathodes of the length of $0.06 \mathrm{~m}$ and $0.164 \mathrm{~m}$, which allows for a detailed study of the effect of the electromagnetic field on the ion current and the process of secondary ion emission. The peak electric potential obtained by solving Eq. (1) was set to return the same value as the anode on the device axis, as noted in [4]. The modeled distribution of ion and electron densities on the midplane are shown in Fig. 2.

The equipotential line and the strength of the generated electric field are presented in Fig. 3. As the image shows, a cathode sheath is formed in the vicinity of the cylindrical cathode, whereas a weak electric field region is formed in about the geometric axis in both, the long and the short cathodes. For the long cathode, the volume of the plasma increases, and the acceleration of the secondary electron emission can be expected as an effect of the magnetic field in the device, which we describe as follows:

The vector potential generated by the circular current

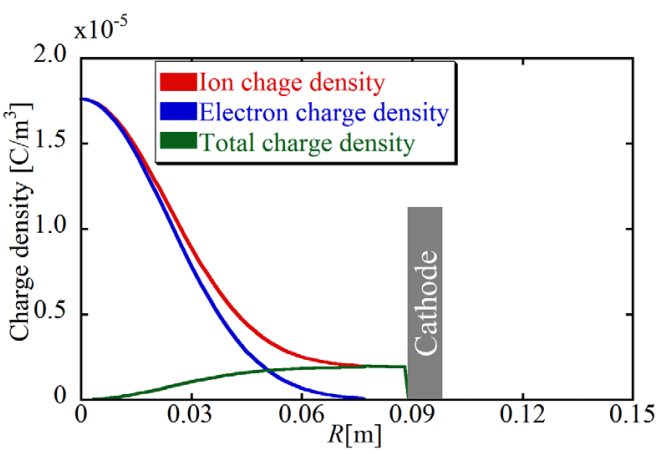

Fig. 2 Ion charge density (red), electron charge density (blue), and total charge density (green).

(a) Cathode length: $0.0600[\mathrm{~m}]$

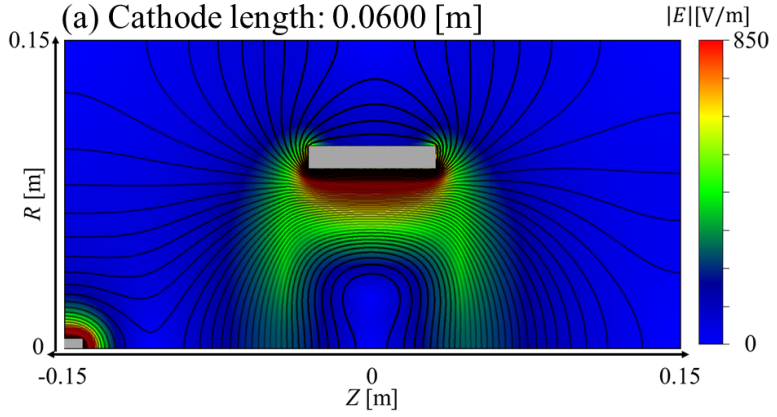

(b) Cathode length: $0.164[\mathrm{~m}]$

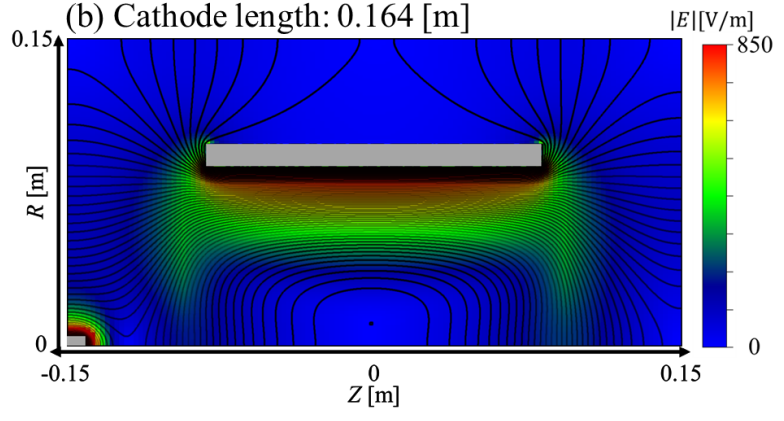

Fig. 3 Electric field structure for the hollow cathode. The color in the graph indicates the strength of the electric field, with the upper limit of $|E|=850 \mathrm{~V} / \mathrm{m}$. The electrode is shown in gray.

$I$ at a radius $a$ is equal to

$$
A_{\theta}=\frac{\mu_{0} I}{4 \pi} \int_{0}^{2 \pi} \frac{a \cos \chi \mathrm{d} \chi}{\sqrt{Z^{2}+R^{2}+a^{2}-2 a R \cos \chi}}
$$

where $\mu_{0}$ is the vacuum permeability. The integral in (3) cannot be solved analytically; hence, we employ numerical analysis to solve the integral. By applying (3) to the magnetic coils, we are able to determine the vector potential produced by each of the coils used. The vector potential created by the solenoid coil and the Helmholtz coil is reproduced by superimposing the obtained vector potentials. To calculate the magnetic field in the device from the 


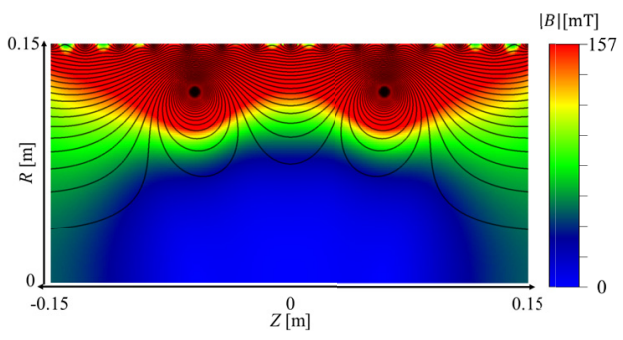

Fig. 4 Magnetic field structure for $I_{\mathrm{s}}=5.0 \mathrm{kA}$ and $I_{\mathrm{h}}=28.6 \mathrm{kA}$. The color indicates the strength of the magnetic field, with the upper limit of $|B|=157 \mathrm{mT}$.

reproduced vector potential, the relation

$$
\mathbf{B}=\operatorname{rot} \mathbf{A},
$$

was used.

For the purpose of this study, the value of the current flowing through the solenoid coil is varied from $0.1 \mathrm{kA}$ to $10.0 \mathrm{kA}$, and the current of the Helmholtz coil is changed accordingly. The generated magnetic field strength and the iso-flux can be seen in Fig. 4, where the solenoid coil current is equal to $I_{\mathrm{S}}=5.0 \mathrm{kA}$ and the Helmholtz coil current is equal to $I_{\mathrm{h}}=28.6 \mathrm{kA}$.

A wide weak magnetic field region is formed in the central part of the device due to the Helmholtz coil. In contrast to the electric field structure shown in Fig. 3, the magnetic force lines penetrate the cathode. As a result, low energy ions are guided to the cathode, where they contribute to the promotion of secondary electron emission, while the ions that have a large momentum in the axial direction can be expected to be drawn out of the magnetic field.

We investigated the single particle trajectory in the electrostatic and magnetic fields formed by a hollow cathode, a solenoid and a Helmholtz coil. It was confirmed that by changing the magnetic field by the solenoid coil, the ratio of loss of deuterium ions and passage in the axial direction in the device wall changes.

\section{Trajectory Calculation}

Deuterium ions within the electric field in the hollow cathode and the magnetic field created by the external field coil move according to the following relations:

$$
\begin{aligned}
& \frac{\mathrm{d} \mathbf{v}}{\mathrm{d} t}=\frac{q}{m}(\mathbf{E}+\mathbf{v} \times \mathbf{B}), \\
& \frac{\mathrm{d} \mathbf{x}}{\mathrm{d} t}=\mathbf{v} .
\end{aligned}
$$

The symbols $\mathbf{x}$ and $\mathbf{v}$ represent the position and the velocity of the deuterium ion, respectively, and the symbols $q$ and $m$ represent the charge and the mass of the deuterium ion, respectively. The solutions to the equations of motion are calculated numerically, using the Runge-Kutta method. We assumed the initial arrangement of deuterium ions to be
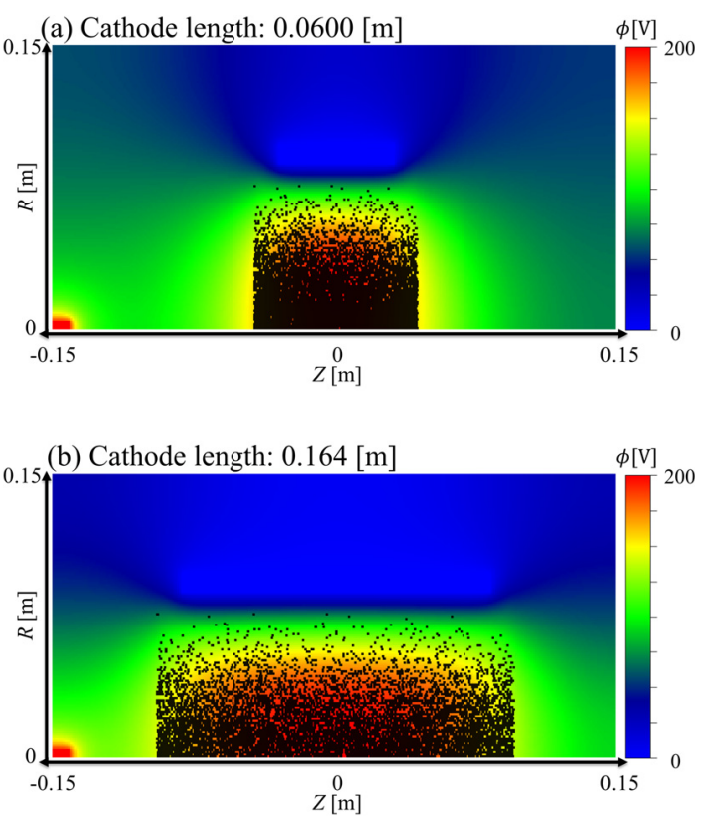

Fig. 5 Initial position of deuterium ions and color contour of the electrostatic potential for the cathode length of (a) $0.06 \mathrm{~m}$ and (b) $0.164 \mathrm{~m}$.

generated as a consequence of ionization by electron collisions, and we set it to correspond to the electron density by the use of pseudorandom numbers. Deuterium gas is thermally moving at room temperature and is four orders of magnitude smaller than electrostatic energy, so the initial velocity of all deuterium ions is assumed to be zero. The obtained initial arrangement of deuterium ions for 10,000 particles is shown in Fig. 5.

The deuterium particles that reached $Z=0.15 \mathrm{~m}$ at the end of the device were regarded as passing particles, whereas the particles that reached the cathode were regarded as lost at the cathode. In addition, the particles that reached $R=0.15 \mathrm{~m}$ were regarded as lost on the device wall.

\section{Results and Discussion}

An example of trajectory calculation result for $I_{\mathrm{s}}=$ $5.0 \mathrm{kA}$ and $I_{\mathrm{h}}=28.6 \mathrm{kA}$ is shown in Fig. 6 .

As can be seen by comparing with the magnetic lines of force in Fig. 4, it can be seen that the magnetic lines exist to wind around the Helmholtz coil. Those particles that collide with the cathode correspond to particles that gyrate around those magnetic force lines. We have also confirmed that the particles near the axis of the device travel straight in the axial direction. Figures 7 and 8 show the histograms of the final energy at the device edge of the passing particles and the energy at the loss in the particles lost at the cathode.

As a result of investigating the passing particles, it was impossible to confirm particles having energy of $200 \mathrm{eV}$ from Figs. 5 and 6. As can be seen from the potential 

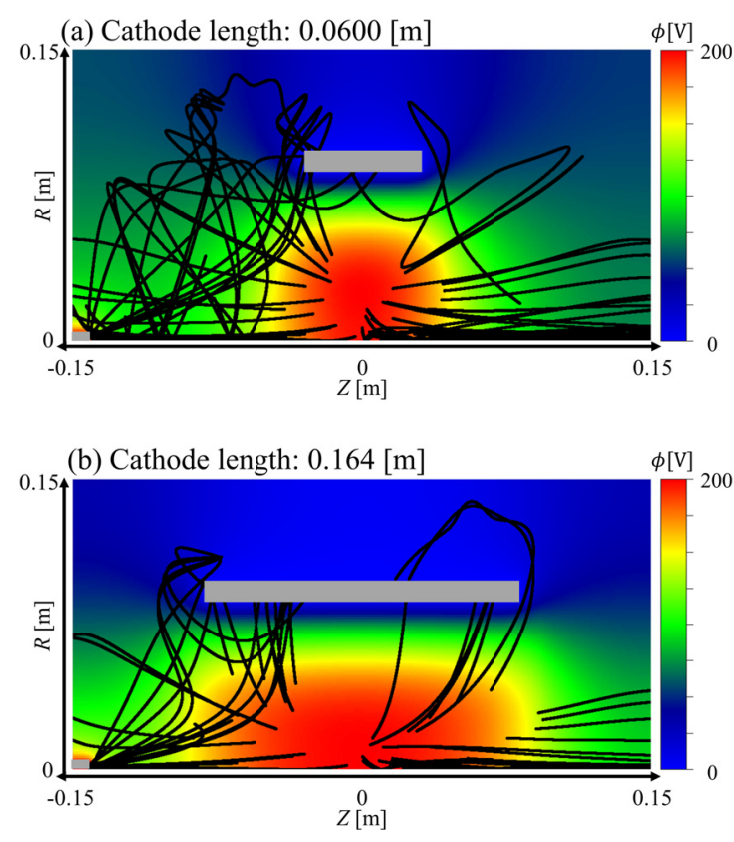

Fig. 6 Typical particle trajectories. (Gray part indicates the electrode.)
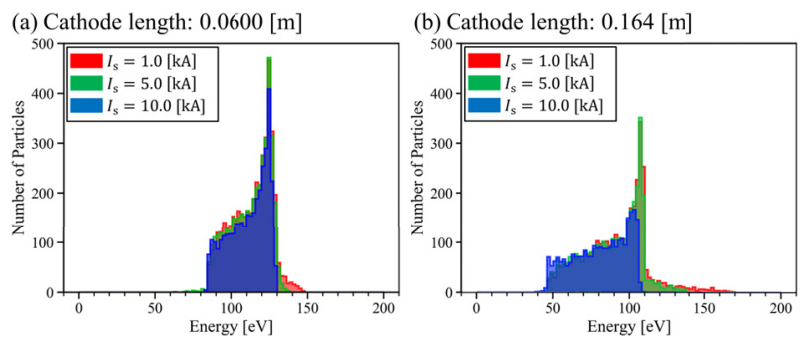

Fig. 7 Energy distribution of passing particles for a cathode length of (a) $0.06 \mathrm{~m}$ and (b) $0.164 \mathrm{~m}$.

distribution in Figs. 5 and 6, this shows that the potential difference between the ion generation region and the device end has not reached $200 \mathrm{~V}$. Moreover, it is considered that most particles having energy of $200 \mathrm{eV}$ collide with the cathode and are lost. In addition, it was found that the maximum value of energy of passing particles is decreased by increasing the solenoid coil current. This is due to the potential distribution that decreases in the radial direction from the axis at the device end and the radial dispersion of the ions decreases as the magnetic field becomes stronger. In order to accelerate the particles to $200 \mathrm{eV}$, it would be necessary to spread them radially at the end of the device in order to obtain a potential difference. We have found that the particles lost at the cathode have energy between $100 \mathrm{eV}$ to $200 \mathrm{eV}$, with many reaching values close to $200 \mathrm{eV}$. We conclude that the deuterium ions are sufficiently accelerated in the cathode sheath.

As Fig. 8 confirms, the fraction of the particles lost at the cathode increases. It is thought that this is because the

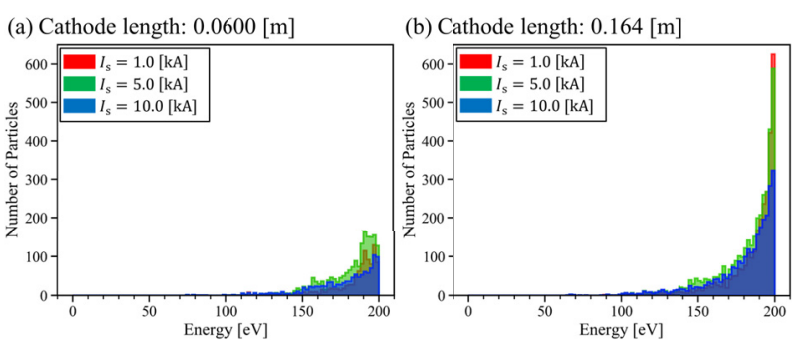

Fig. 8 Energy distribution of particles lost on the cathode with a length of (a) $0.06 \mathrm{~m}$ and (b) $0.164 \mathrm{~m}$.

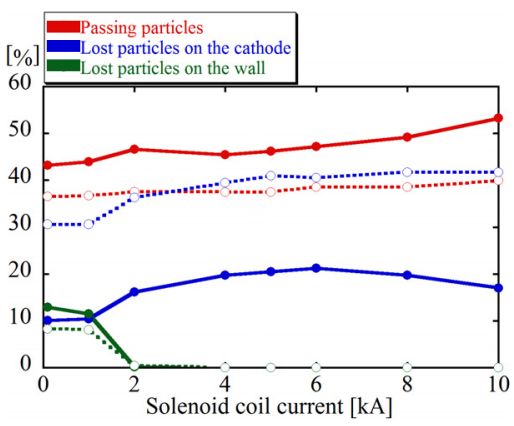

Fig. 9 Distribution of particle types. The solid and dashed lines indicate cathode lengths of $0.06 \mathrm{~m}$ and $0.164 \mathrm{~m}$, respectively.

magnetic force lines in the radial direction formed by the Helmholtz coil are more likely to penetrate the cathode and the particles moving along the magnetic force lines in the radial direction collide with the cathode.

The effects of variation of the solenoid coil current on the ratio of the passing particles, particles lost at the cathode, and those lost at the device is shown in Fig. 9. We have found that, when the length of the cathode is $0.06 \mathrm{~m}$, by increasing the solenoid coil current from $0.1 \mathrm{kA}$ to $10 \mathrm{kA}$, the fraction of the passing particles will increase by $10.1 \%$. Furthermore, it was found that the fraction of passing particles increases by $3.40 \%$ when the length of the cathode is $0.164 \mathrm{~m}$. Also, when the length of the cathode is $0.06 \mathrm{~m}$, increasing the solenoid coil current from $0.1 \mathrm{kA}$ to $10 \mathrm{kA}$ increases the fraction of particles lost at the cathode by $6.90 \%$. And for the length of the cathode to $0.164 \mathrm{~m}$, the increment was found to be $11.1 \%$.

When the solenoid coil current is set to $2.0 \mathrm{kA}$, the fraction of the particles lost at the wall of the device is $0.2 \%$ for the cathode length of $0.06 \mathrm{~m}$ and $0.4 \%$ for $0.164 \mathrm{~m}$, which is a small value. When the solenoid coil current is larger than $2.0 \mathrm{kA}$, the particles lost at the wall of the device cannot be observed. Thus, it is possible to suppress the loss of particles on the device wall by using a solenoid coil current of no less than $2.0 \mathrm{kA}$, and it is possible to increase the number of particles both colliding with the cathode and passing through the device. We predict that the frequency of occurrence of the secondary electron emission can be 
increased by increasing the number of particles colliding with the cathode.

\section{Summary}

We investigated the single particle trajectory in the electrostatic and magnetic fields formed by a hollow cathode, a solenoid and a Helmholtz coil. It was confirmed that by changing the magnetic field by the solenoid coil, the ratio of loss of deuterium ions and passage in the axial direction in the device wall changes.

[1] H. Momota et al., J. Fusion Energy 28, 191 (2009).

[2] Y. Kondoh et al., Phys. Rev. E 63, 036401 (2001).

[3] A. von Engel, Ionized Gases (AIP Press, New York, 1994) p. 218.

[4] Z. Donko, Phys. Rev. E 57, 6 (1998). 